\title{
On Statistical Properties of Fuzzy Random Vectors of Type II *
}

\author{
Tokuo Fukuda
}

\author{
Faculty of Management, Otemon Gakuin University, \\ 2-1-15 Nishi-Ai, Ibaraki, Osaka, 567-8502, JAPAN \\ Phone: Int.+81(Japan)-726-41-9704 \\ Fax: Int.+81(Japan)-726-43-9459 \\ E-Mail: fukuda@res.otemon.ac.jp
}

\begin{abstract}
The author has proposed a kind of fuzzy random vectors(FRVCs in short) which may be appropriate for modeling the data with vagueness and randomness simultaneously[1]. Those FRVCs are considered as the vague perceptions of random phenomena. The purpose of this paper is to propose another type of FRVCs which are not vague perceptions of random phenomena but vague themselves. This another type of FRVCs may be appropriate for modeling human feelings, economic conditions and so on, whose typical properties are that they are obtained as the synthetical verbal expressions of complex random phenomena.

First, using the set representation approach, the reasonable definition of fuzzy random variables(FRVCs) is proposed. Secondly, The statistical moments for the proposed FRVCs and their estimates are investigated. Finally, some of estimates are proposed for the statistical moments of FRVCs.
\end{abstract}

Keywords: fuzzy random data, fuzzy random vectors, set representations of fuzzy sets

\section{Set Representation Approach}

In this paper, a fuzzy set $\widetilde{U}$ is defined by the triple

$$
\widetilde{U}=\left(\mathrm{X},\left\{\widetilde{U}_{\alpha} \mid \alpha \in(0,1)\right\}, s_{\widetilde{U}}\right),
$$

where $\mathrm{X}$ is called the basic space; $s_{\tilde{U}}$ is the predicate, i.e., $s_{\tilde{U}}: \mathrm{X} \rightarrow \mathcal{P}$ with $\mathcal{P}$ the "universe of discourse" defined by a set of statements, assigns a statement

$$
s_{\tilde{U}}(x)=\{x \text { is included in } \widetilde{U}\}
$$

to each element $x \in \mathbf{X}$; and $\left\{\widetilde{U}_{\alpha} \mid \alpha \in(0,1)\right\}$ is the family of subsets of $\mathrm{X}$ satisfying

$$
L_{\alpha} \widetilde{U} \subseteq \widetilde{U}_{\alpha} \subseteq L_{\bar{\alpha}} \widetilde{U} \text { for } \forall \alpha \in(0,1) .
$$

*This work is partly supported by the Ministry of Education, Science, Sports and Culture under Grant-in-Aid for Scientific Re$\operatorname{search}(\mathrm{C})$ No.11680461
In (1.3), $L_{\alpha} \widetilde{U}$ and $L_{\bar{\alpha}} \widetilde{U}$ are the strong cut and the level set of $\widetilde{U}$ at the level $\alpha$ defined respectively by $L_{\alpha} \widetilde{U}=\{x \mid(\widetilde{U})(x)>\alpha\}$ and $L_{\bar{\alpha}} \widetilde{U}=\{x \mid(\widetilde{U})(x) \geq \alpha\}$, where $(\widetilde{U})(x)$ is the membership function of $\widetilde{U}$ given by $(\widetilde{U})(x)=t\left(s_{\widetilde{U}}(x)\right)$ and $t(*)$ is the truth function of * in the sense of multi-valued logic. We call the family of subsets $\left\{\widetilde{U}_{\alpha} \mid \alpha \in(0,1)\right\}$ defined by (2.3) the set representation of a fuzzy set $\widetilde{U}$.

By the way, the author has been proposed another type fuzzy set[2, 3, 1], where instead of (1.2) the predicate $s_{u}$ of (1.1) is given by

$$
s_{\tilde{U}}(x)=\left\{x \text { coincides with } u_{0}\right\}
$$

and $u_{\circ}$ in (1.4) is called original point. The fuzzy set with the predicate given through (1.4) is considered as the vague perception of the crisp point $u_{\mathrm{o}}$, whereas that with (1.2) is vague itself because of, for instance, the synthetical verbal expression of the complex phenomena. For convenience of reference, the fuzzy sets defined in this paper are called those of type II, whereas those defined as vague perceptions of crisp point called those of type I. Hereafter, the types of fuzzy sets are attached only when some confusions will be afraid.

Let the basic space $\mathrm{X}$ be a metric space and let $\left\{\widetilde{U}_{\alpha} \mid \alpha \in(0,1)\right\}$ be the set representation of $\widetilde{U}$. Then, the collection of all fuzzy sets in $\mathrm{X}$ is denoted by $\mathbb{G}(\mathrm{X})$ when it satisfies (i) $\bigcap_{\alpha \in(0,1)} \widetilde{U}_{\alpha} \neq \emptyset$, (ii) $\widetilde{U}$ is compact for each $\alpha \in(0,1)$, and (iii) supp. $\widetilde{U}$ defined by $\operatorname{supp} . \widetilde{U}=\operatorname{cl} . \bigcup_{\alpha \in(0,1)} \widetilde{U}_{\alpha}$ is also compact. The family of fuzzy sets is denoted by $\mathbb{G}_{c}(\mathrm{X})$ when $\widetilde{U}$ is the member of $\mathbb{G}(\mathrm{X})$ and its set representation is composed of convex sets. When $\mathrm{X}$ is the $n$-dimensional Euclidean space $\mathrm{R}^{n}$, the fuzzy sets in $\mathbb{G}_{c}\left(\mathrm{R}^{n}\right)$ is called ( $n$-dimensional) fuzzy vectors and called fuzzy numbers with $n=1$. Hereafter, fuzzy vectors will be represented by lowercase letters with tilde like $\widetilde{u}, \widetilde{v}$, etc. d 


\section{Operations on Fuzzy Sets}

For the arithmetic operations among fuzzy sets of Type II, the same properties are confirmed as those for fuzzy ones of Type I. They are summarized in this section. Let $f: \mathrm{X}_{1} \times \mathrm{X}_{2} \times \cdots \times \mathrm{X}_{n} \rightarrow \mathrm{Y}$ be a mapping from a product metric space $\mathrm{X}_{i}(i=1,2, \cdots, n)$ to the metric space $\mathrm{Y}$. Then, the image of $A_{i} \in \mathrm{X}_{i}(i=1,2, \cdots, n)$ is the subset of $Y$ defined by

$$
\begin{aligned}
f(A)=\{y \in \mathrm{Y} \mid & \mid y=f\left(x_{1}, \cdots, x_{n}\right) \\
& \text { with } \left.x_{i} \in A_{i}(i=1,2, \cdots, n)\right\} .
\end{aligned}
$$

Suppose that $\widetilde{U}_{i}=\left(\mathrm{X},\left\{\widetilde{U}_{i, \alpha} \mid \alpha \in(0,1)\right\}, s_{\tilde{U}_{i}}\right)(i=$ $1,2, \cdots, n)$ are the fuzzy sets, and consider the statement

$$
\begin{aligned}
& s_{f\left(\tilde{U}_{1}, \cdots, \tilde{U}_{n}\right)}(y) \\
& =\left\{y=f\left(x_{1}, \cdots, x_{n}\right) ; x_{i} \in \tilde{U}_{i}(i=1, \cdots, n)\right\} .
\end{aligned}
$$

Then, the above statement should be rewritten as the following composite statement:

$$
\begin{aligned}
s_{f\left(\tilde{U}_{1}, \cdots, \tilde{U}_{n}\right)}(y)= & \bigvee_{x_{i} \in \mathrm{X}_{i}(i=1, \cdots, n)}\left\{\left(y=f\left(x_{1}, \cdots, x_{n}\right)\right)\right. \\
& \left.\wedge\left(\bigwedge_{i=1}^{n}\left(s_{\tilde{U}_{i}}\left(x_{i}\right)\right)\right)\right\},
\end{aligned}
$$

where $s_{\tilde{U}_{i}}\left(x_{i}\right)$ is the statement given by (1.2). Therefore, according to the concept of the extension principle of fuzzy set proposed by Zadeh[4], it may be reasonable to evaluate the truth value of $s_{f\left(\widetilde{U}_{1}, \cdots, \widetilde{U}_{n}\right)}(y)$ as follows:

$$
\begin{aligned}
& t\left(s_{f\left(\tilde{U}_{1}, \cdots, \tilde{U}_{n}\right)}(y)\right) \\
= & \sup _{x_{i} \in \mathrm{X}_{i}(i=1, \cdots, n)}\left\{\min _{i=1, \cdots, n}\left(\widetilde{U}_{i}\right)\left(x_{i}\right) \mid y=f\left(x_{1}, \cdots, x_{n}\right)\right\}
\end{aligned}
$$

with $\sup \emptyset=0$.

Definition 2.1. The fuzzy function $f\left(\widetilde{U}_{1}, \cdots, \widetilde{U}_{n}\right)$ as the generalization of the mapping $f: \mathrm{X}_{1} \times \cdots \times \mathrm{X}_{n} \rightarrow \mathrm{Y}$ is given by

$$
\begin{aligned}
& f\left(\widetilde{U}_{1}, \cdots, \widetilde{U}_{n}\right) \\
& =\left(\mathrm{Y},\left\{f\left(\widetilde{U}_{1, \alpha}, \cdots, \widetilde{U}_{n, \alpha}\right) \mid \alpha \in(0,1)\right\}, s_{f\left(\widetilde{U}_{1}, \cdots, \widetilde{U}_{n}\right)}\right),
\end{aligned}
$$

where $s_{f\left(\widetilde{U}_{1}, \cdots, \widetilde{U}_{n}\right)}$ is the predicate associated with the statement given by (2.2); and $\widetilde{U}_{i, \alpha}$ is the element of the set representation of $\widetilde{U}_{i}$ for each $i=1, \cdots, n$.

It is easy to show

$$
\begin{aligned}
L_{\alpha} f\left(\tilde{U}_{1}, \cdots, \tilde{U}_{n}\right) & \subseteq f\left(L_{\alpha} \widetilde{U}_{1}, \cdots, L_{\alpha} \widetilde{U}_{n}\right) \\
\subseteq f\left(\widetilde{U}_{1, \alpha}, \cdots, \widetilde{U}_{n, \alpha}\right) & \subseteq f\left(L_{\bar{\alpha}} \widetilde{U}_{1}, \cdots, L_{\bar{\alpha}} \widetilde{U}_{n}\right) \\
& \subseteq L_{\bar{\alpha}} f\left(\widetilde{U}_{1}, \cdots, \widetilde{U}_{n}\right) .
\end{aligned}
$$

Theorem 2.1. Let $\left\{\widetilde{U}_{i}=\left(\mathrm{X}_{i},\left\{\widetilde{U}_{i, \alpha} \mid \alpha \in(0,1)\right\}, s_{\widetilde{U}_{i}}\right)\right.$; $i=1, \cdots, n\}$ be a collection of fuzzy sets in $\mathbb{G}\left(\mathrm{X}_{i}\right)$, and $f: \mathrm{X}_{1} \times \cdots \times \mathrm{X}_{n} \rightarrow \mathrm{Y}$ be a mapping from the product space $\mathrm{X}_{1} \times \cdots \times \mathrm{X}_{n}$ of the metric space $\mathrm{X}_{i}$ to the metric space $\mathrm{Y}$. Then,

$$
\begin{aligned}
& f\left(\widetilde{U}_{1}, \widetilde{U}_{2}, \ldots, \widetilde{U}_{n}\right) \\
& \quad=\left(\mathrm{Y},\left\{f\left(\widetilde{U}_{1, \alpha}, \cdots, \widetilde{U}_{n, \alpha}\right) \mid \alpha \in(0,1)\right\}, s_{f\left(\widetilde{U}_{1}, \cdots, \widetilde{U}_{n}\right)}\right)
\end{aligned}
$$

is the fuzzy set satisfying

$$
L_{\alpha} f\left(\widetilde{U}_{1}, \cdots, \widetilde{U}_{n}\right)=f\left(L_{\alpha} \widetilde{U}_{1}, \cdots, L_{\alpha} \widetilde{U}_{n}\right)
$$

for $\forall \alpha \in[0,1)$. Furthermore, if $f$ is a continuous mapping, $f\left(\widetilde{U}_{1}, \cdots, \widetilde{U}_{n}\right)$ is the element of $\mathbb{G}(Y)$ and

$$
L_{\bar{\alpha}} f\left(\widetilde{U}_{1}, \cdots, \widetilde{U}_{n}\right)=f\left(L_{\bar{\alpha}} \widetilde{U}_{1}, \cdots, L_{\bar{\alpha}} \widetilde{U}_{n}\right)
$$

for $\forall \alpha \in(0,1]$.

Let $\widetilde{x}$ be an $n$-dimensional fuzzy vector. Then, as a kind of fuzzy version of the matrix $x x^{\prime}$ generated by the ordinary $n$-dimensional vector $x$, the fuzzy set $\widetilde{x} \square \widetilde{x}^{\prime}$ generated by $\tilde{x}$ is given as follows:

$$
\widetilde{x} \boxminus \widetilde{x}^{\prime}=\left(\mathrm{R}^{n \times n},\left\{\widetilde{x}_{\alpha} \boxminus \widetilde{x}_{\alpha}^{\prime}\right\}, s_{\widetilde{x} \square \widetilde{x}^{\prime}}\right),
$$

where $\widetilde{x}_{\alpha}$ is the element of the set representation of $\widetilde{x}$;

$$
\widetilde{x}_{\alpha} \boxminus \widetilde{x}_{\alpha}^{\prime}=\left\{W \mid W=x x^{\prime}, x \in \widetilde{x}_{\alpha}\right\}
$$

and

$$
s_{\widetilde{x} \boxminus \widetilde{x}^{\prime}}(W)=\left\{W \text { is included in } \widetilde{x} \widetilde{x}^{\prime}\right\} .
$$

Furthermore, for the two fuzzy vectors $\widetilde{x}$ and $\widetilde{y}$, as a kind of fuzzy version of the matrix $x y^{\prime}$, the fuzzy set $\widetilde{x} \cdot \widetilde{y}^{\prime}$ is given as follows:

$$
\widetilde{x} \cdot \widetilde{y}^{\prime}=\left(\mathrm{R}^{n \times m},\left\{\widetilde{x}_{\alpha} \cdot \widetilde{y}_{\alpha}^{\prime}\right\}, s \widetilde{x} \cdot \widetilde{y}^{\prime}\right),
$$

where $\widetilde{x}_{\alpha}$ and $\widetilde{y}_{\alpha}$ are the elements of the set representations of $\widetilde{x}$ and $\widetilde{y}$, respectively;

$$
\widetilde{x}_{\alpha} \cdot \widetilde{y}_{\alpha}^{\prime}=\left\{W \mid W=x y^{\prime}, x \in \widetilde{x}_{\alpha}, y \in \widetilde{y}_{\alpha}\right\}
$$

and

$$
s_{\widetilde{x}} \cdot \widetilde{y}^{\prime}(W)=\left\{W \text { is included in } \widetilde{x} \widetilde{y}^{\prime}\right\} .
$$

Then, applying Theorem 2.1 to (2.10) and (2.13), we have the following Corollary:

Corollary 2.1. Let $\tilde{x} \in \mathbb{G}_{c}\left(\mathrm{R}^{n}\right)$ and $\tilde{y} \in \mathbb{G}_{c}\left(\mathrm{R}^{m}\right)$ be the fuzzy vectors. Let $\left\{\widetilde{x}_{\alpha} \mid \alpha \in(0,1)\right\}$ and $\left\{\widetilde{x}_{\alpha} \mid \alpha \in\right.$ $(0,1)\}$ be their set representations, respectively. Then, 
the fuzzy set given by (2.10) and (2.13) are fuzzy matrices, i.e.,

$$
\widetilde{x} \square \widetilde{x}^{\prime} \in \mathbb{G}\left(\mathrm{R}^{n \times n}\right) \text { and } \widetilde{x} \cdot \widetilde{y}^{\prime} \in \mathbb{G}\left(\mathrm{R}^{n \times m}\right) \text {, }
$$

which satisfy

$$
\begin{aligned}
L_{\alpha}\left(\widetilde{x} \boxminus \widetilde{x}^{\prime}\right) & =\left(L_{\alpha} \widetilde{x}\right) \square\left(L_{\alpha} \widetilde{x}\right)^{\prime} \\
L_{\alpha}\left(\widetilde{x} \cdot \widetilde{y}^{\prime}\right) & =\left(L_{\alpha} \widetilde{x}\right) \cdot\left(L_{\alpha} \widetilde{y}\right)^{\prime} \\
L_{\bar{\alpha}}\left(\widetilde{x} \square \widetilde{x}^{\prime}\right) & =\left(L_{\bar{\alpha}} \widetilde{x}\right) \square\left(L_{\bar{\alpha}} \widetilde{x}\right)^{\prime}
\end{aligned}
$$

and

$$
L_{\bar{\alpha}}\left(\widetilde{x} \cdot \widetilde{y}^{\prime}\right)=\left(L_{\bar{\alpha}} \widetilde{x}\right) \cdot\left(L_{\bar{\alpha}} \widetilde{y}\right)^{\prime} .
$$

Furthermore, the following relation is also confirmed:

$$
\widetilde{x} \square \widetilde{x}^{\prime} \subseteq \widetilde{x} \cdot \widetilde{x}^{\prime} .
$$

\section{$3 \quad$ Fuzzy Random Vectors}

Let $(\Omega, \mathfrak{A}, P)$ be a probability space, where $\mathfrak{A}$ is the $\sigma$ algebra generated by the subsets of $\Omega$, and $P$ is a probability measure. In order to define FRVCs, let $\mathfrak{K}_{c}^{n}$ be the family of all the non-empty compact convex subsets of $\mathrm{R}^{n}$, and $\mathfrak{B}\left(\mathfrak{K}_{c}^{n}\right)$ stands for the minimum $\sigma$-algebra generated by the open subsets of $\mathfrak{K}_{c}^{n}$, where the topology of $\mathfrak{K}_{c}^{n}$ is generated by the Hausdorff metric.

Definition 3.1. The FRVC $\widetilde{x}$ is defined by

$$
\tilde{x}: \Omega \rightarrow \mathbb{G}_{c}\left(\mathrm{R}^{n}\right)
$$

with

$$
\widetilde{x}(\omega)=\left(R^{n},\left\{\widetilde{x}_{\alpha}(\omega) \mid \alpha \in(0,1)\right\}, s_{\widetilde{x}}\right),
$$

where $s_{\widetilde{x}}$ is the predicate associated with the statement such as

$$
s_{\widetilde{x}}(x, \omega)=\{x \in \widetilde{x}(\omega)\},
$$

its set representation $\left\{\widetilde{x}_{\alpha} \mid \alpha \in(0,1)\right\}$ is composed of $\mathfrak{A}$-measurable compact and convex correspondences from $\Omega$ to $\mathrm{R}^{n}[5]$. The measurability of $\widetilde{x}$ is given through that of the function $\widetilde{x}_{\alpha}$ from $\Omega$ to $\mathfrak{B}\left(\mathfrak{K}_{c}^{n}\right)$.

Let $\widetilde{x}$ be a FRVC. Assume that there exists $h(\omega)$ such that

$$
\left\|\xi_{\alpha}(\omega)\right\| \leq h(\omega) \text { a.s. }
$$

for each $\xi_{\alpha} \in \widetilde{x}_{\alpha}$ a.s. and $\alpha \in(0,1)$, where $h(\omega)$ is the random variable such that

$$
E\left\{h^{p}\right\}=\int h^{p} d P<\infty .
$$

The FRVC satisfying (3.5) for any $\alpha \in(0,1)$ is called $L^{p}$-integrably bounded. It is easy to see that the random variable $\xi_{\alpha}$ included in $\widetilde{x}_{\alpha}$ almost surely satisfies

$$
\xi_{\alpha} \in L^{p}(\Omega, P)=\left\{\left.\xi\left|\int\right| \xi\right|^{p} d P<+\infty\right\}
$$

when $\widetilde{x}_{\alpha}$ is $L^{p}$-integrably bounded.

\section{Expectations of FRVCs}

In order to investigate the statistical properties of FRVCs, let $\widetilde{x}$ be a $L^{1}$-integrably bounded FRV. Consider first the statement given by

$$
s_{E\{\tilde{x}\}}(x)=\{x \in \text { the expectation of } \widetilde{x}\} .
$$

Then, it may be considered that above statement is given as a composite statement such as

$$
s_{E\{\tilde{x}\}}(x)=\bigvee_{\xi \in L^{1}(\Omega, P)}\{(x=E\{\xi\}) \wedge(\xi \in \widetilde{x} \text { a.s. })\} \text {. }
$$

Furthermore, we can consider that the statement $(\xi \in$ $\widetilde{x}$ a.s.) in (4.2) is given by

$$
(\xi \in \widetilde{x} \text { a.s. })=\bigwedge_{\omega \in \Omega \backslash N_{0}} s_{\tilde{x}}(\xi, \omega),
$$

where $\mathrm{N}_{0}$ is the null set, i.e., $P\left(\mathrm{~N}_{0}\right)=0$ with $\mathrm{N}_{0} \in \mathfrak{A}$, and $s \tilde{x}(\xi, \omega)$ is the statement given by (3.3). Then, (4.2) is represented by

$$
\begin{aligned}
& s_{E\{\tilde{x}\}}(x) \\
& =\bigvee_{\xi \in L^{1}(\Omega, P)}\left\{(x=E\{\xi\}) \wedge\left(\bigwedge_{\omega \in \Omega \backslash N_{0}} s_{\tilde{x}}(\xi, \omega)\right)\right\} .
\end{aligned}
$$

Therefore, applying the concept of the extension principle for fuzzy sets, it may be considered that the truth value of $s_{E\{\tilde{x}\}}(x)$ is given by

$$
\begin{aligned}
& t\left(s_{E\{\tilde{x}\}}(x)\right) \\
& =\sup _{\xi \in L^{1}(\Omega, P)}\left\{\operatorname{essinf}_{\omega \in \Omega} t\left(s_{\widetilde{x}}(\xi, \omega)\right) \mid x=E\{\xi\}\right\},
\end{aligned}
$$

where $\operatorname{essinf}_{\omega \in \Omega} t\left(s_{\widetilde{x}}(\xi, \omega)\right)$ is given by the supremum of $a$ satisfying $t\left(s_{\widetilde{x}}(\xi, \omega)\right)=(\widetilde{x}(\omega))(\xi) \geq a$ almost surely. We introduce here the Aumann integral[6] given by

$$
\text { (A) } \int \widetilde{x}_{\alpha} d P=\left\{x \mid x=\int \xi d P, \quad \xi \in S\left(\widetilde{x}_{\alpha}\right)\right\},
$$

where $\widetilde{x}_{\alpha}$ is arbitrary element of the set representation of $\widetilde{x}$, and $S\left(\widetilde{x}_{\alpha}\right)$ is the selection set of $\widetilde{x}_{\alpha}$, i.e.,

$$
S\left(\widetilde{x}_{\alpha}\right)=\left\{\xi \mid \xi \in L^{1}(\Omega, P) ; \xi(\omega) \in \widetilde{x}_{\alpha}(\omega) \text { a.s. }\right\} .
$$

Using Aumann integral given above, the expectation of $\widetilde{x}_{\alpha}$ is defined by

$$
E\left\{\widetilde{x}_{\alpha}\right\}=(\mathrm{A}) \int \widetilde{x}_{\alpha} d P .
$$

When $\widetilde{x}$ is $L^{1}$-integrably bounded, it can be shown that $\widetilde{x}_{\alpha}$ is integrable for each $\alpha \in(0,1)$, i.e., $E\left\{\widetilde{x}_{\alpha}\right\} \neq \emptyset[6]$. 
Proposition 4.1. Let $\widetilde{x} \in \mathbb{G}_{c}\left(R^{n}\right)$ be a $L^{1}$-integrably bounded FRVC and $\left\{\widetilde{x}_{\alpha} \mid \alpha \in(0,1)\right\}$ be its set representation. Then,

$$
\begin{aligned}
\left\{x \mid t\left(s_{E\{\tilde{x}\}}(x)\right)\right. & >\alpha\} \subseteq E\left\{\widetilde{x}_{\alpha}\right\} \\
& \subseteq\left\{x \mid t\left(s_{E\{\tilde{x}\}}(x)\right) \geq \alpha\right\}
\end{aligned}
$$

for $\forall \alpha \in(0,1)$.

Therefore, the following definition of the expectation of a FRVC $\widetilde{x}$ may be reasonable:

Definition 4.1. Let $\widetilde{x}$ be a $L^{1}$-integrably bounded FRVC and $\left\{\widetilde{x}_{\alpha} \mid \alpha \in(0,1)\right\}$ be its set representation. Then, the expectation of the FRVC $\widetilde{x}$ is defined by

$$
E\{\widetilde{x}\}=\left(\mathrm{R},\left\{E\left\{\widetilde{x}_{\alpha}\right\} \mid \alpha \in(0,1)\right\}, s_{E\{\widetilde{x}\}}\right),
$$

where $s_{E\{\widetilde{x}\}}$ is the predicate associated with the statement given by (4.1); and $\left\{E\left\{\tilde{x}_{\alpha}\right\} \mid \alpha \in(0,1)\right\}$ is the set representation of $E\{\widetilde{x}\}$ given through (4.8).

It can be also shown that

$$
L_{\alpha} E\{\widetilde{x}\} \subseteq E\left\{L_{\alpha} \widetilde{x}\right\} \subseteq E\left\{\widetilde{x}_{\alpha}\right\} \subseteq E\left\{L_{\bar{\alpha}} \widetilde{x}\right\} \subseteq L_{\bar{\alpha}} E\{\widetilde{x}\} .
$$

\section{Second Moments of FRVCs}

As shown in Corollary 2.1, $\widetilde{x} \boxminus \widetilde{x}^{\prime}$ is a fuzzy matrix when $\widetilde{x}$ is a fuzzy vector. Therefore, it is possible to consider the second moment of $\widetilde{x}$. Let $\widetilde{x}$ be a $L^{2}$-integrably bounded FRVC. Then, the statements like

$$
s_{E\left\{\widetilde{x} \boxminus \widetilde{x}^{\prime}\right\}}(x)=\left\{x \in E\left\{\widetilde{x} \boxminus \widetilde{x}^{\prime}\right\}\right\}
$$

and

$$
s_{\text {var. }} \widetilde{x}(x)=\{x \in \text { variance of } \widetilde{x}\}
$$

are rewritten by

$$
\begin{aligned}
& s_{E\left\{\tilde{x} \boxminus \widetilde{x}^{\prime}\right\}}(x) \\
& =\bigvee_{\xi \in L^{2}(\Omega, P)}\left\{\left(x=E\left\{\xi \xi^{\prime}\right\}\right) \wedge\left(\bigwedge_{\omega \in \Omega \backslash N_{0}} s_{\widetilde{x}}(\xi, \omega)\right)\right\}
\end{aligned}
$$

and

$$
\begin{aligned}
& s_{\text {var. }} \widetilde{x}(x) \\
= & \bigvee_{\xi \in L^{2}(\Omega, P)}\left\{(x=\text { var. } \xi) \wedge\left(\bigwedge_{\omega \in \Omega \backslash N_{0}} s_{\widetilde{x}}(\xi, \omega)\right)\right\}
\end{aligned}
$$

respectively, where $s_{\widetilde{x}}(\xi, \omega)$ is the statement given by (3.3), and

$$
\text { var. } \xi=E\left\{(\xi-E\{\xi\})(\xi-E\{\xi\})^{\prime}\right\} .
$$

Then, using same procedure as that in Sec. 4, we can define second statistical moments of the FRVC $\widetilde{x}$.
Definition 5.1. Let $\widetilde{x} \in \mathbb{G}_{c}\left(\mathrm{R}^{n}\right)$ be an $L^{2}$-integrably bounded FRVC and $\left\{\widetilde{x}_{\alpha} \mid \alpha \in(0,1)\right\}$ be its set representation. Then, the second moment $E\left\{\widetilde{x} \square \widetilde{x}^{\prime}\right\}$ of $\widetilde{x}$ is defined by

$E\left\{\widetilde{x} \boxminus \widetilde{x}^{\prime}\right\}=\left(\mathrm{R}^{n \times n},\left\{E\left\{\widetilde{x}_{\alpha} \boxminus \widetilde{x}_{\alpha}^{\prime}\right\} \mid \alpha \in(0,1)\right\}, s_{E\left\{\tilde{x} \boxminus \widetilde{x}^{\prime}\right\}}\right)$,

where $s_{E\left\{\tilde{x} \square \tilde{x}^{\prime}\right\}}$ is the predicate associated with the statement given through (5.1); and $\left\{E\left\{\widetilde{x}_{\alpha} \square \widetilde{x}_{\alpha}^{\prime}\right\} \mid \alpha \in\right.$ $(0,1)\}$ is the set representation of $E\left\{\widetilde{x} \square \widetilde{x}^{\prime}\right\}$. Furthermore, the variance of $\widetilde{x}$ is defined by

$$
\text { var. } \widetilde{x}=\left(\mathrm{R}^{n \times n},\left\{\text { var. } \widetilde{x}_{\alpha} \mid \alpha \in(0,1)\right\}, s_{\text {var. }} \tilde{x}\right) \text {, }
$$

where $s_{\text {var. }} \widetilde{x}$ is the predicate associated with the statement given by (5.2); and $\left\{\right.$ var. $\left.\widetilde{x}_{\alpha} \mid \alpha \in(0,1)\right\}$ is the set representation of var. $\widetilde{x}$ given through

$$
\text { var. } \widetilde{x}_{\alpha}=E\left\{\left(\widetilde{x}_{\alpha} \boxminus E\left\{\widetilde{x}_{\alpha}\right\}\right)\left(\widetilde{x}_{\alpha} \boxminus E\left\{\widetilde{x}_{\alpha}\right\}\right)^{\prime}\right\}
$$

and

$$
\begin{aligned}
\widetilde{x}_{\alpha} & \boxminus E\left\{\widetilde{x}_{\alpha}\right\} \\
& =\left\{x \mid x=\xi-E\{\xi\}, \xi \in L^{2}(\Omega, P), \xi \in \widetilde{x}_{\alpha}\right\} .
\end{aligned}
$$

Definition 5.2. Let $\widetilde{x} \in \mathbb{G}_{c}\left(\mathrm{R}^{n}\right)$ be a $L^{2}$-integrably bounded FRVC and $\left\{\widetilde{x}_{\alpha} \mid \alpha \in(0,1)\right\}$ be its set representation. Then, the pseudo-variance of $\widetilde{x}$ is defined by

$$
\mathrm{p} \text {-var. } \widetilde{x}=\left(\mathrm{R}^{n \times n},\left\{\mathrm{p} \text {-var. } \widetilde{x}_{\alpha} \mid \alpha \in(0,1)\right\}, s_{\mathrm{p} \text {-var. } \widetilde{x}}\right),
$$

where $s_{\mathrm{p}-\mathrm{var} . \widetilde{x}}$ is the predicate associated with the statement given by

$$
s_{\mathrm{p}-\operatorname{var} . \widetilde{x}}(x)=\left\{x \in E\left\{(\widetilde{x}-E\{\widetilde{x}\}) \square(\widetilde{x}-E\{\widetilde{x}\})^{\prime}\right\}\right\},
$$

$\left\{\mathrm{p}\right.$-var. $\left.\widetilde{x}_{\alpha} \mid \alpha \in(0,1)\right\}$ is the set representation given through

$$
\begin{gathered}
\text { p-var. } \widetilde{x}_{\alpha}=E\left\{\left(\widetilde{x}_{\alpha}-E\left\{\widetilde{x}_{\alpha}\right\}\right) \odot\left(\widetilde{x}_{\alpha}-E\left\{\widetilde{x}_{\alpha}\right\}\right)^{\prime}\right\} \\
=\left\{x \mid x=E\left\{\xi \xi^{\prime}\right\}, \xi=\xi_{1}-E\left\{\xi_{2}\right\},\right. \\
\left.\xi_{i} \in \widetilde{x}_{\alpha}, \xi_{i} \in L^{2}(\Omega, P)(i=1 \text { and } 2)\right\} .
\end{gathered}
$$

It is clear from the definition of p-var. $\widetilde{x}$ that

$$
\text { var. } \widetilde{x} \subseteq \text { p-var. } \widetilde{x}
$$

in the sense of

$$
\text { var. } \widetilde{x}_{\alpha} \subseteq \text { p-var. } \widetilde{x}_{\alpha} \quad \text { for } \forall \alpha \in(0,1) .
$$




\section{Cross Moments of FRVCs}

For the cross moment between FRVCs $\widetilde{x}$ and $\widetilde{y}$, same argument as that in Sec. 5 are valid. Let $\widetilde{x}$ and $\widetilde{y}$ be $L^{2}$-integrably bounded FRVCs. Then, the statements of

$$
s_{E\left\{\widetilde{x} \cdot \widetilde{y}^{\prime}\right\}}(x)=\left\{x \in E\left\{\widetilde{x} \cdot \widetilde{y}^{\prime}\right\}\right\}
$$

and

$$
s_{\text {cov. }}(\tilde{x}, \widetilde{y})(x)=\{x \in \operatorname{cov} .(\widetilde{x}, \widetilde{y})\}
$$

are written by

$$
\begin{aligned}
& s_{E\left\{\tilde{x} \cdot \tilde{y}^{\prime}\right\}}(x)=\bigvee_{\xi, \zeta \in L^{2}(\Omega, P)}\left\{\left(M=E\left\{\xi \zeta^{\prime}\right\}\right)\right. \\
& \left.\wedge\left(\bigwedge_{\omega \in \Omega \backslash N_{0}} s_{\tilde{x}}(\xi, \omega)\right) \wedge\left(\bigwedge_{\omega \in \Omega \backslash N_{0}} s_{\tilde{y}}(\zeta, \omega)\right)\right\},
\end{aligned}
$$

and

$$
\begin{gathered}
s_{\text {cov. }(\tilde{x}, \tilde{y})}(x)=\bigvee_{\xi, \zeta \in L^{2}(\Omega, P)}\{(M=\operatorname{cov} .(\xi, \zeta)) \\
\left.\wedge\left(\bigwedge_{\omega \in \Omega \backslash N_{0}} s_{\tilde{x}}(\xi, \omega)\right) \wedge\left(\bigwedge_{\omega \in \Omega \backslash N_{0}} s \widetilde{y}(\zeta, \omega)\right)\right\}
\end{gathered}
$$

respectively, where $s_{\widetilde{x}}(\xi, \omega)$ is the statement given by (3.3), and

$$
\text { cov. }(\xi, \zeta)=E\left\{(\xi-E\{\xi\})(\zeta-E\{\zeta\})^{\prime}\right\} .
$$

Then, we can define cross moments between $\widetilde{x}$ and $\widetilde{y}$ as follows:

Definition 6.1. Let $\widetilde{x} \in \mathbb{G}_{c}\left(\mathrm{R}^{n}\right)$ and $\widetilde{y} \in \mathbb{G}_{c}\left(\mathrm{R}^{m}\right)$ be $L^{2}$-integrably bounded FRVCs, and $\left\{\widetilde{x}_{\alpha} \mid \alpha \in(0,1)\right\}$ and $\left\{\widetilde{y}_{\alpha} \mid \alpha \in(0,1)\right\}$ be their set representations. Then, the cross moment $E\left\{\widetilde{x} \cdot \widetilde{y}^{\prime}\right\}$ between $\widetilde{x}$ and $\widetilde{y}$ is defined by

$E\left\{\widetilde{x} \cdot \widetilde{y}^{\prime}\right\}=\left(\mathrm{R}^{n \times m},\left\{E\left\{\widetilde{x}_{\alpha} \cdot \widetilde{y}_{\alpha}^{\prime}\right\} \mid \alpha \in(0,1)\right\}, s_{E\left\{\tilde{x} \cdot \tilde{y}^{\prime}\right\}}\right)$,

where $s_{E\left\{\tilde{x} \cdot \tilde{y}^{\prime}\right\}}$ is the predicate associated with the statement given through (6.1); and $\left\{E\left\{\widetilde{x}_{\alpha} \cdot \widetilde{y}_{\alpha}^{\prime}\right\} \mid \alpha \in(0,1)\right\}$ is the set representation of $E\left\{\widetilde{x} \cdot \widetilde{y}^{\prime}\right\}$. Furthermore, the covariance between $\widetilde{x}$ and $\widetilde{y}$ is defined by

cov. $(\widetilde{x}, \widetilde{y})=\left(R^{n \times m},\left\{\operatorname{cov} .\left(\widetilde{x}_{\alpha}, \widetilde{y}_{\alpha}\right) \mid \alpha \in(0,1)\right\}, s_{\text {var. }} \widetilde{x}\right)$,

where $s_{\text {cov. }}(\widetilde{x}, \widetilde{y})$ is the predicate associated with the statement given by (6.2); and $\left\{\right.$ cov. $\left.\left(\widetilde{x}_{\alpha}, \widetilde{y}_{\alpha}\right) \mid \alpha \in(0,1)\right\}$ is the set representation of cov. $(\widetilde{x}, \widetilde{y})$ given through $\operatorname{cov} .\left(\widetilde{x}_{\alpha}, \widetilde{y}_{\alpha}\right)=E\left\{\left(\widetilde{x}_{\alpha} \boxminus E\left\{\widetilde{x}_{\alpha}\right\}\right) \cdot\left(\widetilde{y}_{\alpha} \boxminus E\left\{\widetilde{y}_{\alpha}\right\}\right)^{\prime}\right\}$,

and $\widetilde{x}_{\alpha} \boxminus E\left\{\widetilde{x}_{\alpha}\right\}$ and $\widetilde{y}_{\alpha} \boxminus E\left\{\widetilde{y}_{\alpha}\right\}$ are given by (5.8b).
Definition 6.2. Let $\widetilde{x}$ and $\widetilde{y}$ be $L^{2}$-integrably bounded FRVCs and $\left\{\widetilde{x}_{\alpha} \mid \alpha \in(0,1)\right\}$ and $\left\{\widetilde{y}_{\alpha} \mid \alpha \in(0,1)\right\}$ be their set representations, respectively. Then, the pseudo-covariance between $\widetilde{x}$ and $\widetilde{y}$ is defined by

$$
\begin{aligned}
& \text { p-cov. }(\widetilde{x}, \widetilde{y}) \\
& =\left(\mathrm{R}^{n \times m},\left\{\text { p-cov. }\left(\widetilde{x}_{\alpha}, \widetilde{y}_{\alpha}\right) \mid \alpha \in(0,1)\right\}, s_{\text {p-var. } \widetilde{x}}\right),
\end{aligned}
$$

where $s_{\mathrm{p} \text {-cov. }(\tilde{x}, \tilde{y})}$ is the predicate associated with the statement given by

$s_{\text {p-cov. }(\tilde{x}, \tilde{y})}(M)=\left\{M \in E\left\{(\widetilde{x}-E\{\widetilde{x}\}) \square(\widetilde{y}-E\{\widetilde{y}\})^{\prime}\right\}\right.$,

$\left\{\mathrm{p}\right.$-cov. $\left.\left(\widetilde{x}_{\alpha}, \widetilde{y}_{\alpha}\right) \mid \alpha \in(0,1)\right\}$ is the set representation given through

$$
\text { p-cov. }\left(\widetilde{x}_{\alpha}, \widetilde{y}_{\alpha}\right)=E\left\{\left(\widetilde{x}_{\alpha}-E\left\{\widetilde{x}_{\alpha}\right\}\right) \square\left(\widetilde{y}_{\alpha}-E\left\{\widetilde{y}_{\alpha}\right\}\right)^{\prime}\right\}
$$

Since

$$
\text { cov. } \begin{aligned}
&\left(\widetilde{x}_{\alpha}, \widetilde{y}_{\alpha}\right)=\{M \mid M=E\{(\xi-E\{\xi\}) \\
&\left.\left.\times(\zeta-E\{\zeta\})^{\prime}\right\}, \xi \in \widetilde{x}_{\alpha}, \zeta \in \widetilde{y}_{\alpha}\right\}
\end{aligned}
$$

and

$$
\begin{array}{r}
\text { p-cov. }\left(\widetilde{x}_{\alpha}, \widetilde{y}_{\alpha}\right)=\left\{M \mid M=E\left\{\left(\xi_{1}-E\left\{\xi_{2}\right\}\right)\right.\right. \\
\left.\left.\times\left(\zeta_{1}-E\left\{\zeta_{2}\right\}\right)^{\prime}\right\}, \xi_{1}, \xi_{2} \in \widetilde{x}_{\alpha}, \zeta_{1}, \zeta_{2} \in \widetilde{y}_{\alpha}\right\},
\end{array}
$$

it is clear that

$$
\operatorname{cov} .(\widetilde{x}, \widetilde{y}) \subseteq \mathrm{p}-\operatorname{cov} \cdot(\widetilde{x}, \widetilde{y})
$$

in the sense of

$$
\operatorname{cov} .\left(\widetilde{x}_{\alpha}, \widetilde{y}_{\alpha}\right) \subseteq \text { p-cov. }\left(\widetilde{x}_{\alpha}, \widetilde{y}_{\alpha}\right)
$$

for $\forall \alpha \in(0,1)$.

\section{Moment Estimates of FRVCs}

Let $\widetilde{x}_{i}=\left(\mathrm{R}^{n},\left\{\widetilde{x}_{i, \alpha} \mid \alpha \in(0,1)\right\}, s \widetilde{x}_{i}\right)(i=1, \cdots, n)$ be a sequence of $L^{1}$-integrably bounded FRVCs. Then, as the estimate of the expectation $E\left\{\widetilde{x}_{1}\right\}$, the fuzzy set

$$
\widehat{\widetilde{x}}(N)=\left(\mathrm{R},\left\{(\widehat{\widetilde{x}}(N))_{\alpha} \mid \alpha \in(0,1)\right\}, s_{\widetilde{\widetilde{x}}(N)}\right)
$$

is heuristically given, where the predicate $s_{\widetilde{\widetilde{x}}(N)}$ is given through the statement

$$
s_{\widetilde{\widetilde{x}}(N)}(v)=\left\{v \text { is included in } \frac{1}{N} \sum_{i=1}^{N} \widetilde{x}_{i}\right\} .
$$

Applying Theorem 2.1, we can see that the fuzzy set $\widehat{\widetilde{x}}(N)$ given by $(7.1)$ is a fuzzy number, i.e., $\widehat{\widetilde{x}}(N) \epsilon$ 
$\mathbb{G}_{c}(\mathrm{R})$, and each element of the set representation of $\widehat{\widetilde{x}}(N)$ is given by

$$
(\widehat{\widetilde{x}}(N))_{\alpha}=\frac{1}{N} \sum_{i=1}^{N} \widetilde{x}_{i, \alpha} \quad \text { for } \alpha \in(0,1),
$$

The strong cut and the level set of $\widehat{\widetilde{x}}(N)$ are respectively given by

$$
\begin{array}{ll}
L_{\alpha} \widehat{\widetilde{x}}(N)=\frac{1}{N} \sum_{i=1}^{N} L_{\alpha} \widetilde{x}_{i} \quad \text { for } \alpha \in[0,1), \\
L_{\bar{\alpha}} \widehat{\widetilde{x}}(N)=\frac{1}{N} \sum_{i=1}^{N} L_{\bar{\alpha}} \widetilde{x}_{i} \quad \text { for } \alpha \in(0,1] .
\end{array}
$$

Then, it is reasonable to write

$$
\widehat{\widetilde{x}}(N)=\frac{1}{N} \sum_{i=1}^{N} \widetilde{x}_{i} .
$$

Same discussion as above is valid for the estimates of second statistical moments of $\widetilde{x}$ and also valid for the cross moments between two FRVCs $\widetilde{x}$ and $\widetilde{y}$. Only the results of them are shown below due to the shortage of space.

$$
\begin{gathered}
\widehat{\widetilde{x} \boxminus \widetilde{x}^{\prime}}(N)=\frac{1}{N} \sum_{i=1}^{N} \widetilde{x}_{i} \boxminus \widetilde{x}_{i} \\
\widehat{\text { p-var. } \widetilde{x}(N)}=\frac{1}{N} \sum_{i=1}^{N}\left(\widetilde{x}_{i}-\widehat{\widetilde{x}}(N)\right) \square\left(\widetilde{x}_{i}-\widetilde{\widetilde{x}}(N)\right)^{\prime} \\
\widetilde{\widetilde{x} \cdot \widetilde{y}^{\prime}}(N)=\frac{1}{N} \sum_{i=1}^{N} \widetilde{x}_{i} \cdot \widetilde{y}_{i}^{\prime}
\end{gathered}
$$

and

$$
\begin{aligned}
& \widehat{\operatorname{p-cov} .(\widetilde{x}}, \widetilde{y})(N) \\
& =\frac{1}{N} \sum_{i=1}^{N}\left(\widetilde{x}_{i}-\widehat{\widetilde{x}}(N)\right) \cdot\left(\widetilde{y}_{i}-\widehat{\widetilde{y}}(N)\right)^{\prime}
\end{aligned}
$$

where elements of their set representations are given by

$$
\begin{gathered}
\left(\widehat{\widetilde{x} \square \widetilde{x}^{\prime}}(N)\right)_{\alpha}=\frac{1}{N} \sum_{i=1}^{N} \widetilde{x}_{i, \alpha} \boxminus \widetilde{x}_{i, \alpha}^{\prime} \\
(\widehat{\mathrm{p} \text {-var. } \widetilde{x}}(N))_{\alpha} \\
\quad=\frac{1}{N} \sum_{i=1}^{N}\left(\widetilde{x}_{i, \alpha}-\widehat{\widetilde{x}_{\alpha}}(N)\right) \square\left(\widetilde{x}_{i, \alpha}-\widehat{\widetilde{x}_{\alpha}}(N)\right)^{\prime}
\end{gathered}
$$

$$
\left(\widehat{\widetilde{x} \cdot \widetilde{y}^{\prime}}(N)\right)_{\alpha}=\frac{1}{N} \sum_{i=1}^{N} \widetilde{x}_{i, \alpha} \cdot \widetilde{y}_{i, \alpha}^{\prime}
$$

and

$$
\begin{aligned}
& \widehat{(\mathrm{p}-\widehat{\operatorname{cov} .}(\widetilde{x}}, \widetilde{y})(N))_{\alpha} \\
& \quad=\frac{1}{N} \sum_{i=1}^{N}\left(\widetilde{x}_{i, \alpha}-\widehat{\widetilde{x}_{\alpha}}(N)\right) \cdot\left(\widetilde{y}_{i, \alpha}-\widetilde{\widetilde{y}_{\alpha}}(N)\right)^{\prime}
\end{aligned}
$$

for $\alpha \in(0,1)$.

\section{Conclusions}

In this paper, another kind of FRVCs have been proposed, where the underlying FRVCs are those obtained as, for instance, the verbal expressions of complex random phenomena not the vague perceptions of random phenomena. The some of statistical moments of the proposed FRVCs have been investigated and their estimates have also proposed.

The statistical properties of the proposed FRVCs are under the intensive investigation now from both numerical and theoretical viewpoints and will be reported in the near future.

\section{References}

[1] T. Fukuda. On a class of fuzzy random vectors(in Japanese). Journal of Japan Society for Fuzzy Theory and Systems, 10(3):499-505, 1998.

[2] T. Fukuda. On fuzzy random vectors as vague perceptions of random phenomena and their statistical moments. In The Proceedings of 35th IEEE CDC, pages 2719-2724, 1996.

[3] T. Fukuda. Fuzzy random data obtained as vague perceptions of random phenomena. In T. Katayama and S. Sugimoto, editors, Statistical Methods in Control and Signal Processing, pages 303-330. Marcel Dekker, Inc., 1997.

[4] L. A. Zadeh. The concept of a linguistic variables and its application in approximate reasoning, parts 1, 2 and 3. Information Sciences, 8 and 9:199249,301-357,43-80, respectively, 1975,76.

[5] E. Klein and A. C. Thompson. Theory of Correspondences. John Wiley \& Sons, Netherlands, 1988.

[6] R. J. Aumann. Integrals of set-valued functions. Journal of Mathematical Analysis and its Applications, 12:1-12, 1965. 